\title{
Ferromagnetism in the Kondo-lattice
}

\author{
Philipp Wagner ${ }^{1}$, Piotr Wróbel ${ }^{2}$, and Robert Eder ${ }^{1, a}$ \\ ${ }^{1}$ Karlsruhe Institute of Technology, Institut für Festkörperphysik, 76021 Karlsruhe, Germany \\ ${ }^{2}$ Institute for Low Temperature and Structure Research, P.O. Box 1410, 50-950 Wrocław 2, Poland
}

Received 11 November 2019 / Received in final form 5 February 2020

Published online 1 April 2020

(C) The Author(s) 2020. This article is published with open access at Springerlink.com

\begin{abstract}
We discuss the ferromagnetic phase of the planar Kondo lattice model using the bond fermion technique. For sufficiently small Kondo exchange we find a weakly ferromagnetic phase already for the weakly doped Kondo insulator. We present the phase diagram of the model and its quasiparticle band structure in the different phases.
\end{abstract}

\section{Introduction}

Heavy Fermions are intermetallic compounds containing (mostly) Cerium, Ytterbium or Uranium and are characterized by a paramagnetic low-temperature Fermi liquid phase with the characteristic heavy bands and a Fermi surface volume corresponding to itinerant $4 f$ or $5 f$ electrons $[1,2]$. Many of these compounds show magnetically ordered phases, whereby the ordering transition often is accompanied by a change of the Fermi surface volume, as if the $f$-electrons were to change from itinerant to localized. In many compounds the transition temperature can be tuned down to zero by external parameters resulting in quantum critical points, non-Fermi liquid behaviour and superconducting domes [3-5].

The appropriate model to study Heavy Fermions is the Kondo lattice model (KLM) which in the presence of a magnetic field $\mathbf{B}$ can be written as

$$
\begin{aligned}
H= & \sum_{\mathbf{k}, \sigma} \epsilon_{\mathbf{k}} c_{\mathbf{k}, \sigma}^{\dagger} c_{\mathbf{k}, \sigma}+J \sum_{j} \mathbf{S}_{j} \cdot \sigma_{j} \\
& -\sum_{j} \mathbf{B} \cdot\left(\mathbf{S}_{j}+\sigma_{j}\right) .
\end{aligned}
$$

The model is defined on a lattice (in the present work: a simple cubic planar lattice) of $N$ unit cells, whereby each unit cell $j$ contains one conduction band (or $c$ ) orbital and one localized (or $f$ ) orbital, the operators $c_{j, \sigma}^{\dagger}$ and $f_{j, \sigma}^{\dagger}$ create an electron with z-spin $\sigma$ in these orbitals. Moreover, $\sigma_{j}=\frac{1}{2} c_{j, \sigma}^{\dagger} \boldsymbol{\tau}_{\sigma \sigma^{\prime}} c_{j, \sigma^{\prime}}$, with $\boldsymbol{\tau}$ the vector of Pauli matrices, is the spin operator for conduction electrons, whereas $\mathbf{S}_{j}$ denotes the spin operator for $f$ electrons, defined in an analogous way. We will assume from now on that the magnetic field is along the $z$-direction $\mathbf{B}=B \mathbf{e}_{z}$. An important feature of the model is the constraint to have precisely one

\footnotetext{
${ }^{\mathrm{a}}$ e-mail: robert.eder@kit.edu
}

electron per $f$-orbital:

$$
\sum_{\sigma} f_{j, \sigma}^{\dagger} f_{j, \sigma}=1
$$

which must hold separately for each unit cell $j$ and reflects the nature of the KLM as the strong-coupling limit [6] of the periodic Anderson model (PAM). The number $N_{c}$ of conduction electrons is variable, we denote their density/unit cell as $n_{c}=N_{c} / N$, the total electron density then is $n_{e}=1+n_{c}$. Since we will mostly consider electron densities slightly below 2 we also introduce $\delta=2-n_{e}$. Finally

$$
\epsilon_{\mathbf{k}}=-2 t\left(\cos \left(k_{x}\right)+\cos \left(k_{y}\right)\right)+4 t_{1} \cos \left(k_{x}\right) \cos \left(k_{y}\right)
$$

is the dispersion relation of the conduction band, parameterized by a nearest-neighbor hopping integral $-t$ and 2 nd nearest neighbor hopping integral $t_{1}$.

The KLM has been discussed frequently by using the mean-field (or saddle-point) approximation, where the exchange term which is quartic in electron operators is mean-field factorized [7-17]. There have also been many numerical studies using density matrix renormalization group calculations [18-22], quantum Monte-Carlo [23], series expansion [24,25] variational Monte-Carlo (VMC) [26-29] Dynamical Mean Field Theory (DMFT) [30] or Dynamical Cluster Approximation (DCA) [31,32].

While antiferromagnetic order is common in heavy Fermions, there are also some ferromagnetic compounds such as $\mathrm{UGe}_{2}$, URhGe, UCoGe [33] or UCoAl [34]. These materials have received considerable attention due to the coexistence of superconductivity and ferromagnetism [35]. Ferromagnetism in the KLM or PAM was studied in the mean-field approximation [36], the Gutzwiller wave function [37-40], VMC [28,29], DMFT [41-43] and DCA [43]. In the present manuscript we want to explore 
the possibility of ferromagnetic ordering in the KLM by using bond fermion theory [44-46]. It was found previously $[47,48]$ that for the antiferromagnetic phases, bond fermion theory agrees well with numerical results for the 2D KLM. More precisely, the quite intricate phase diagram in the $\left(J / t, n_{c}\right)$ plane obtained from bond Fermion theory agrees qualitatively with that from VMC $[26,27,29]$ and DMFT [30], the quasiparticle band structure for the antiferromagnetic phase of the Kondo insulator - i.e. the KLM for $n_{e}=2$ - and its change with $J$ agree in considerable detail with that from DCA [32]. The main deficiency is that bond Fermion theory appears to have problems to reproduce energy scales correctly. For example, bond fermion theory finds the value of $J_{c}$ below which antiferromagnetic order sets in for the Kondo insulator with $t_{1}=0$ to be $J_{c}=2.3$ [47], whereas the exact value is $J_{c}=1.45$ [23]. This is probably not too servere a drawback in that even numerical methods have problems to determine $J_{c}$ correctly: VMC finds $J_{c}=1.7[26]$, DMFT finds $J_{c}=2.2$ [30] and DCA gives $J_{c}=2.1$ [32]. However, these deficiencies have to be kept in mind.

\section{Formalism}

The basic idea of the bond fermion formalism is to introduce a mapping between a subspace of the Hilbert space for the true KLM Hamiltonian to the Hilbert space of a fictitious system of noninteracting fermions. More precisely, we define

$$
\tilde{s}_{j}^{\dagger}=\frac{\cos (\Theta)}{\sqrt{2}}\left(c_{j, \uparrow}^{\dagger} f_{j, \downarrow}^{\dagger}-c_{j, \downarrow}^{\dagger} f_{j, \uparrow}^{\dagger}\right)+\sin (\Theta) c_{j, \uparrow}^{\dagger} f_{j, \uparrow}^{\dagger}
$$

and

$$
\left|\Psi_{0}\right\rangle=\prod_{j=1}^{N} \tilde{s}_{j}^{\dagger}|0\rangle
$$

The state $\tilde{s}_{j}^{\dagger}|0\rangle$ is a superposition of a singlet and the triplet with $S_{z}=1$, whereby the degree of admixture of the triplet is determined by the angle $\Theta$. The expectation value of $H$ in the state $\left|\Psi_{0}\right\rangle$ is $-N \tilde{e}_{0}$ with

$$
-\tilde{e}_{0}=-\frac{3 J}{4} \cos ^{2}(\Theta)+\left(\frac{J}{4}-B\right) \sin ^{2}(\Theta) .
$$

The expectation value of $S_{z}$ is $N \tilde{m}_{0}$ with $\tilde{m}_{0}=\frac{1}{2} \sin (2 \Theta)$. For $\Theta \neq 0$ the state $\left|\Psi_{0}\right\rangle$ thus has a nonvanishing ferromagnetic polarization which may be viewed as resulting from the condensation of triplets into momentum $(0,0)$ [49] on a background of singlets.

From now on we consider $\left|\Psi_{0}\right\rangle$ as the vacuum state of the bond fermion Hilbert space. The bond fermions themselves are defined as follows: if the cell $j$ is in the state $f_{j, \sigma}^{\dagger}|0\rangle$ we consider it as occupied by a hole-like Fermion, created by $a_{j, \sigma}^{\dagger}$, in the bond fermion Hilbert space, whereas if the cell is in the state $c_{j, \uparrow}^{\dagger} c_{j, \downarrow}^{\dagger} f_{j, \sigma}^{\dagger}|0\rangle$ we consider it as occupied by an electron-like Fermion, created by $b_{j, \sigma}^{\dagger}$. We denote the set of cells occupied by a single electron (three electrons) by $S_{a}\left(S_{b}\right)$ and define $S_{s}$ as the complement of $S_{a} \cup S_{b}$ (i.e. $S_{s}$ is the set of cells with two electrons). The correspondence between the bond fermion states and the states of the KLM is

$$
\begin{aligned}
& \left(\prod_{i \in S_{a}} a_{i, \sigma_{i}}^{\dagger}\right)\left(\prod_{j \in S_{b}} b_{j, \sigma_{j}}^{\dagger}\right)|0\rangle \\
& \rightarrow\left(\prod_{i \in S_{a}} f_{i, \sigma_{i}}^{\dagger}\right)\left(\prod_{j \in S_{b}} c_{j, \uparrow}^{\dagger} c_{j, \downarrow}^{\dagger} f_{j, \sigma_{j}}^{\dagger}\right)\left(\prod_{n \in S_{s}} \tilde{s}_{n}^{\dagger}\right)|0\rangle .
\end{aligned}
$$

It is obvious that in order for this mapping to make sense the sets of sites $S_{a}$ and $S_{b}$ have to be disjunct, which is equivalent to an infinitely strong repulsion between the bond particles $a_{j, \sigma}^{\dagger}$ and $b_{j, \sigma}^{\dagger}$ in the same cell $j$. However, as discussed in reference [47], the density of the $a^{\dagger}$ and $b^{\dagger}$ Fermions is quite small for $J / t>1$ and $\delta \leq 0.3$ so that the constraint can be relaxed to good approximation.

To transcribe operators for the physical KLM into 'bond fermion language' we demand that their matrix elements between the bond Fermion states on the left hand side of (6) are equal to those of the true KLM operator between the corresponding KLM states on the right hand side. Due to the product nature of the KLM states these matrix elements are easy to evaluate. For example, the electron creation operators, from which many operators can be constructed, become

$$
\begin{aligned}
& c_{j, \uparrow}^{\dagger}=-\frac{\cos (\Theta)}{\sqrt{2}}\left(b_{j, \uparrow}^{\dagger}-a_{j, \downarrow}\right)+\sin (\Theta) a_{j, \uparrow}, \\
& c_{j, \downarrow}^{\dagger}=-\frac{\cos (\Theta)}{\sqrt{2}}\left(b_{j, \downarrow}^{\dagger}+a_{j, \uparrow}\right)-\sin (\Theta) b_{j, \uparrow}^{\dagger} .
\end{aligned}
$$

Upon Fourier transform and introducing the operator column vector $\mathbf{v}_{\mathbf{k}}=\left(b_{\mathbf{k}, \uparrow}, a_{-\mathbf{k}, \downarrow}^{\dagger}, b_{\mathbf{k}, \downarrow}, a_{-\mathbf{k}, \uparrow}^{\dagger}\right)^{T}$ we find the bond Fermion representation of the kinetic energy

$$
H_{t}=\sum_{\mathbf{k}, \sigma} \epsilon_{\mathbf{k}} c_{\mathbf{k}, \sigma}^{\dagger} c_{\mathbf{k}, \sigma} \rightarrow \sum_{\mathbf{k}} \mathbf{v}_{\mathbf{k}}^{\dagger} H_{\mathbf{k}} \mathbf{v}_{\mathbf{k}}
$$

whereby

$$
H_{\mathbf{k}}=\frac{\epsilon_{\mathbf{k}}}{2}\left(\begin{array}{rrr}
c_{1}, & -c_{2}, c_{3}, & 0 \\
-c_{2}, & c_{2}, & 0, c_{3} \\
c_{3}, & 0, c_{2}, c_{2} \\
0, & c_{3}, c_{2}, c_{1}
\end{array}\right)
$$

with

$$
\begin{aligned}
& c_{1}=1+\sin ^{2}(\Theta), \\
& c_{2}=\cos ^{2}(\Theta) \\
& c_{3}=\sqrt{2} \sin (\Theta) \cos (\Theta) .
\end{aligned}
$$


The exchange and magnetic-field terms in (1) become

$$
\begin{aligned}
H_{J}= & J \sum_{j} \mathbf{S}_{j} \cdot \sigma_{j}-\sum_{j} \mathbf{B} \cdot\left(\mathbf{S}_{j}+\sigma_{j}\right) \\
\rightarrow & \sum_{j, \sigma}\left(\tilde{e}_{0}-\frac{B}{2} \operatorname{sign}(\sigma)\right)\left(b_{j, \sigma}^{\dagger} b_{j, \sigma}+a_{j, \sigma}^{\dagger} a_{j, \sigma}\right) \\
& -N \tilde{e}_{0} .
\end{aligned}
$$

The number of electrons - including the $f$-electrons can be expressed as follows: the vacuum state $\left|\Psi_{0}\right\rangle$ - see (4) - contains $2 N$ electrons. Since a hole-like bond Fermions reduces this by one and each electron-like Fermion adds an additional electron we have

$$
\begin{aligned}
N_{e} & =2 N+\sum_{\mathbf{k}, \sigma}\left(b_{\mathbf{k}, \sigma}^{\dagger} b_{\mathbf{k}, \sigma}-a_{\mathbf{k}, \sigma}^{\dagger} a_{\mathbf{k}, \sigma}\right) \\
& =\sum_{\mathbf{k}, \sigma}\left(b_{\mathbf{k}, \sigma}^{\dagger} b_{\mathbf{k}, \sigma}+a_{\mathbf{k}, \sigma} a_{\mathbf{k}, \sigma}^{\dagger}\right) .
\end{aligned}
$$

Adding up the terms above we can write down $H_{B F}-\mu N$ which obviously is quadratic in the bond fermion operators - this is the simplification due to the bond Fermion formulation and this is possible because the exchange term (12) can be written as a quadratic form. Setting $\Theta=0$ the $4 \times 4$ matrix $H_{\mathbf{k}}$ in (9) decomposes into two independent blocks of dimension $2 \times 2$, which differ only in the sign of the off-diagonal element $c_{2}$. This gives the four quasiparticle energies

$$
E_{\mathbf{k}, \sigma, \pm}=\frac{\epsilon_{\mathbf{k}}}{2} \pm \sqrt{\tilde{e}_{0}^{2}+\left(\frac{\epsilon_{\mathbf{k}}}{2}\right)^{2}}-\frac{B}{2} \operatorname{sign}(\sigma)-\mu,
$$

which correspond to the mere spin-splitting of the bands obtained for $B=0$. For $\Theta \neq 0$ there will be an additional deformation of these bands, so that the bond fermion formalism does not just correspond to a spin splitting of the heavy bands.

The $f$-electron and $c$-electron magnetization per site, $m_{f}=\left\langle S_{i, z}\right\rangle$ and $m_{c}=\left\langle\sigma_{i, z}\right\rangle$ become

$$
\begin{aligned}
m_{f}= & \frac{1}{N} \sum_{j, \sigma}\left(\frac{1}{2} \operatorname{sign}(\sigma)-\tilde{m}_{0}\right)\left(b_{j, \sigma}^{\dagger} b_{j, \sigma}+a_{j, \sigma}^{\dagger} a_{j, \sigma}\right) \\
& -2 \tilde{m}_{0}, \\
m_{c}= & -\frac{\tilde{m}_{0}}{N} \sum_{j, \sigma}\left(b_{j, \sigma}^{\dagger} b_{j, \sigma}+a_{j, \sigma}^{\dagger} a_{j, \sigma}\right)-2 \tilde{m}_{0} .
\end{aligned}
$$

These expressions show that a ferromagnetic polarization of the system can come about in two ways: the first term becomes nonzero due to a polarization of the bond Fermion bands, the additive constant becomes nonzero due to the ferromagnetic polarization of the 'singlet background'. For small $\tilde{m}_{0}$ - which will be seen to be realized in most cases - the $c$-electron polarization is much weaker than that of the $f$-electron. This is understandable from the definition of the $a_{j}^{\dagger}$ and $b_{j}^{\dagger}$ Fermions which correspond to either 0 or $2 c$-electrons in cell $j$-put another way, the spin of the bond Fermions is always carried by the $f$-electron.

A complication of the bond Fermion calculations which has been discussed previously $[44,45,47]$ is the fact that without further provisions the $\mathbf{k}$ - and $\omega$-integrated spectral weight in the $c$-electron Green's function is not equal to $N_{c}$. The reason simply is the small spectral weight of the 'heavy' band portions. As discussed previously, this can be fixed by enforcing the consistency by an additional constraint with Lagrange multiplier $\lambda$. The consequence of this is the replacement $\epsilon_{\mathbf{k}} \rightarrow \epsilon_{\mathbf{k}}-\lambda$ in (9) and an additional additive constant $+\lambda N_{c}$ in the Hamiltonian $[44,45,47]$.

Once we relax the constraint of no double occupancy implied by (6) the approximate $H-\mu N$ can be readily diagonalized by a unitary transformation and we obtain four bands, denoted by $E_{\nu, \mathbf{k}}, \nu=1 \ldots 4$, and the resulting quasiparticle operators $\gamma_{\mathbf{k}, \nu}^{\dagger}$. Since the transformation from the $b_{\mathbf{k}, \sigma}^{\dagger}$ and $a_{-\mathbf{k}, \bar{\sigma}}$ to the $\gamma_{\mathbf{k}, \nu}^{\dagger}$ is unitary, the electron number (13) becomes

$$
N_{e}=\sum_{\mathbf{k}, \nu} \gamma_{\mathbf{k}, \nu}^{\dagger} \gamma_{\mathbf{k}, \nu}
$$

which shows that in the bond Fermion formalism the $f$ electrons always participate in the Fermi surface volume despite their strictly localized nature.

Moreover is obvious from (9) that the eigenstates of $H_{B F}$ are mixtures of spin- $\uparrow$ and spin- $\downarrow$ bond particles and thus do not have a definite $z$-spin. Namely the first two components of the vector $\mathbf{v}_{\mathbf{k}}$ obey $\left[S_{z}, b_{\mathbf{k}, \uparrow}\right]=$ $\left[S_{z}, a_{-\mathbf{k}, \downarrow}^{\dagger}\right]=-\frac{1}{2}$, whereas the two last terms obey $\left[S_{z}, b_{\mathbf{k}, \downarrow}\right]=\left[S_{z}, a_{-\mathbf{k}, \uparrow}^{\dagger}\right]=\frac{1}{2}$. In the quasiparticle operators $\gamma_{\mathbf{k}, \nu}$ these terms are mixed due to the presence of the matrix elements $\propto c_{3}$ in (9). Similarly, the bond Fermion vacuum $\left|\Psi_{0}\right\rangle$ in (4) also does not have a definite $z$-spin. On the other hand, the weight of components with different $z$-spin will be peaked around $\sum_{i}\left\langle m_{f}+m_{c}\right\rangle$ with a relative width that vanishes in the thermodynamical limit so that this is not a problem.

Knowing the band structure one can calculate the ground state energy $E_{0}$ as a function of the as yet undetermined angle $\Theta$ in (4). $\Theta$ then is determined by minimizing $E_{0}$ as a function of $\Theta$. Since we are working with a discrete $\mathrm{k}$-mesh and since it turns out that for the ferromagnetic case very small energy differences are involved we actually work at a small but finite temperature and instead minimize the Helmholtz free energy

$$
F=-\frac{1}{\beta} \sum_{\mathbf{k}, \nu} \log \left(1+e^{-\beta E_{\nu, \mathbf{k}}}\right)+\mu N_{e}+\lambda N_{c}+N \tilde{e}_{0}
$$

We choose $T=0.004 t$ which results in smooth $F(\Theta)$ curves for a k-mesh with stepsize $\frac{\pi}{100}$.

Equation (6) shows the main advantage of bond fermion theory, namely all basis states fulfil the constraint (2) exactly. On the other hand, it is obvious from the above 


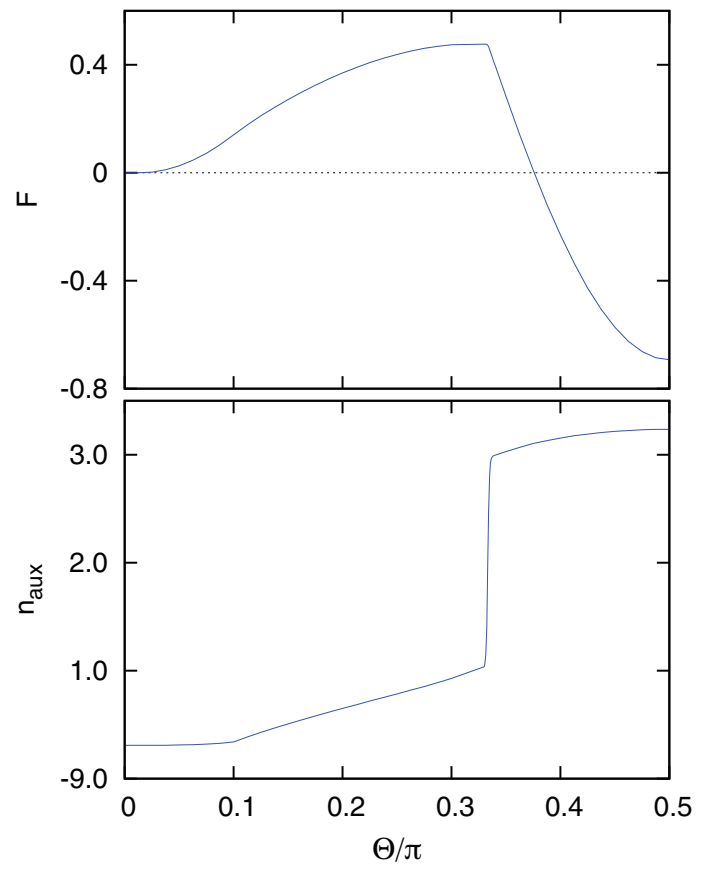

Fig. 1. Free energy (top) and density of bond fermions per site (bottom) versus $\Theta$ for $J / t=2.3, t_{1}=0, n_{e}=1.9$. The free energy for $\Theta=0$ has been chosen as the zero of energy.

that bond Fermion theory is by nature a strong coupling theory so that one cannot expect it to reproduce the energy scale of the single impurity Kondo temperature $T_{K}=W e^{-1 / \rho J}$, which emerges in the limit of small $J / t$ ( $W$ and $\rho$ are the bandwidth and density of states of the conduction band). In fact, for $J / t \rightarrow 0$ the density of the fermions increases strongly [47] so that relaxing the constraint of no double occupancy cannot be expected to be a meaningful approximation anymore.

Further consideration shows that the bond Fermion formalism cannot describe a fully polarized ferromagnetic state, which would correspond to $\Theta=\frac{\pi}{2}$. This can be seen from Figure 1 which shows $F$ as a function of $\Theta$. $F$ initially increases with $\Theta$, but then bends down sharply at $\Theta=\frac{\pi}{3}$ and has a pronounced minimum at $\Theta=\frac{\pi}{2}$. At first glance this appears to indicate that the ground state is the fully polarized ferromagnetic state but this is incorrect. This can be seen from the lower part of the figure, which shows the density of the bond Fermions

$$
n_{a u x}=\frac{1}{N} \sum_{\mathbf{k}, \sigma}\left\langle b_{\mathbf{k}, \sigma}^{\dagger} b_{\mathbf{k}, \sigma}+a_{\mathbf{k}, \sigma}^{\dagger} a_{\mathbf{k}, \sigma}\right\rangle
$$

as a function of $\Theta$. While this relatively small, $n_{\text {aux }} \approx$ 0.3 , at small $\Theta$, it increases with increasing $\Theta$ and then jumps discontinuously to $n_{\text {aux }} \approx 3$ at $\Theta=\frac{\pi}{3}$. Since the bond Fermions have to obey the constraint that no two of them can occupy the same site, their density/site cannot exceed 1 for any physical state. A bond Fermion state with $n_{\text {aux }} \approx 3$ therefore cannot possibly represent a state of the Kondo lattice and the minimum at $\Theta=\frac{\pi}{2}$ is unphysical. The reason for the jump of $n_{\text {aux }}$ at $\Theta \stackrel{2}{=} \frac{\pi}{3}$ is that for
$B=0$ the energy of a bond fermion, $\tilde{e}_{0}$, becomes negative (see Eq. (5)). It therefore becomes energetically favourable for the system to create bond fermions, which is possible due to terms like $a_{m, \uparrow}^{\dagger} b_{n, \downarrow}^{\dagger}$, so that the system is 'flooded with fermions'. This problem is easily fixed, however, by restricting the range of $\Theta$ to be between 0 and the angle $\Theta_{1}$, where the Fermion density/site exceeds 1 . It turns out that the minima of $F$ always occur for $\Theta \ll \Theta_{1}$ so this is more of a formal restriction.

The failure of the bond particle formalism to describe the fully polarized state is no surprise because by construction it can only describe a state where the $f$-electrons participate in the Fermi surface volume, see (16). To investigate the possibility of the $f$-electrons 'dropping out' of the Fermi surface we need to estimate the energy of the strongly polarized state where the $f$-electrons do not contribute to the Fermi surface volume. We define

$$
\left|\Psi_{0}\right\rangle=\prod_{j} f_{j, \uparrow}^{\dagger}|0\rangle \otimes|F S\rangle,
$$

where $|F S\rangle$ denotes the (spin-polarized) Fermi sea for the conduction electrons. The Fermi energy thereby is calculated from the constraint that the total number of $c$-electrons be $N_{c}$. The exchange term becomes $\frac{J}{2} \sum_{j} \sigma_{j}^{z}$ and the quasiparticle bands and Free energy are

$$
\begin{aligned}
E_{\mathbf{k}, \sigma} & =\epsilon_{\mathbf{k}} \mp \frac{B}{2} \pm \frac{J}{4}, \\
F & =-\frac{1}{\beta} \sum_{\mathbf{k}, \sigma} \log \left(1+e^{E_{\mathbf{k}, \sigma}-\mu}\right)-\frac{N B}{2} .
\end{aligned}
$$

It is clear that this is a crude estimate so that comparison of the energy with the Kondo state described by the bond Fermions has a rather limited significance.

\section{Results}

We first consider the Kondo state as described by the bond Fermion theory at $B=0$. Figure 2 shows the Helmhotz free energy $F$ as a function of $\Theta$ for different values of $J$, whereby $\delta=0.1, t_{1}=0$. To facilitate the comparison of the curves, the respective value of $F$ for $\Theta=0$ has been chosen as the zero of energy for each $J$. For $J=2.5$ and $J=2.4$ the minimum of $F$ is obtained for $\Theta=0$ so that the ground state is paramagnetic (the figure shows only a small range of $\Theta$ but for larger $\Theta$ in fact $F$ increases monotonously up to $\Theta_{1}$ ). For the smaller values $J=2.3$ and $J=2.2$ there is a minimum for a nonvanishing albeit small $\Theta$ indicating a spontaneous ferromagnetic polarization. Apparently there is a rapid increase of $\Theta$ from zero to a value around $0.01 \pi$ in a small range of $J$ around $J=2.35$. The lowering of $F$ due to the ferromagnetic polarization is quite small, however, of order $10^{-4} t$.

Figure 3 shows the angle $\Theta$ which minimizes $F$ as a function of $J$ for different electron densities and $t_{1}=0$. Also shown is the $f$-like magnetization $m_{f}$, see equation (15) 


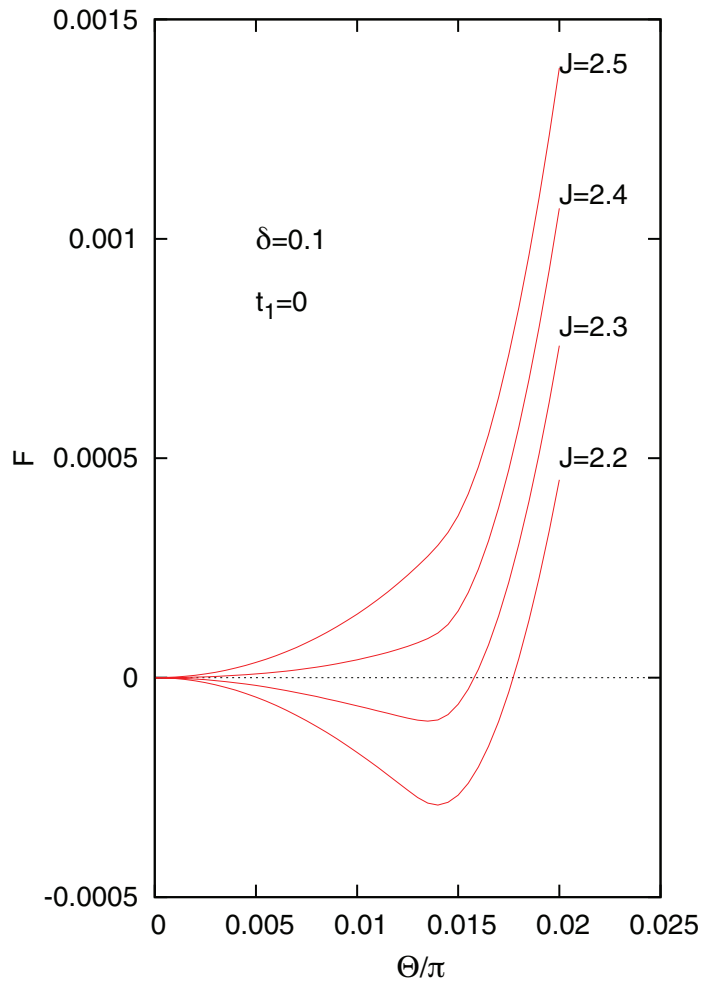

Fig. 2. Helmhotz Free energy as a function of the angle $\Theta$ for different $J$ and $t_{1}=0.0$.

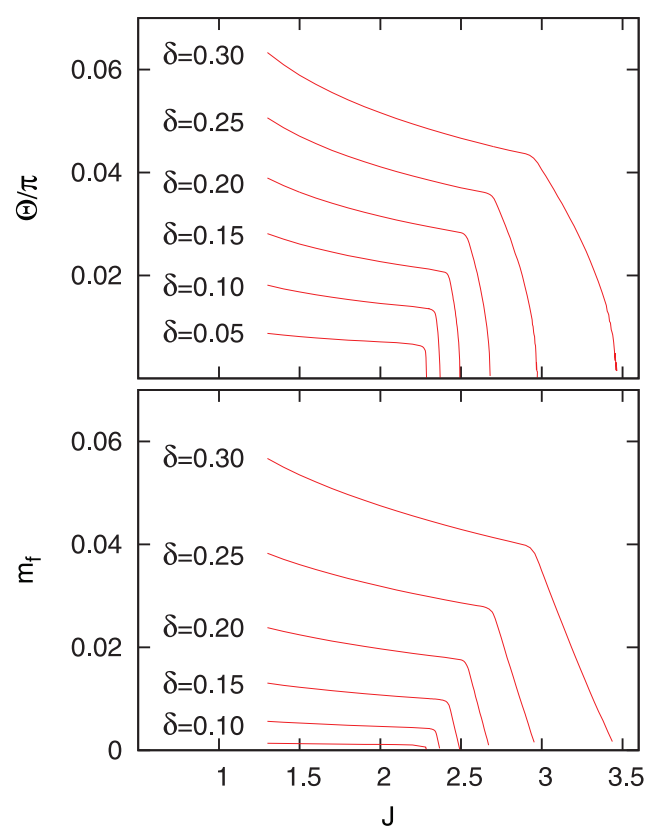

Fig. 3. Angle $\Theta$ which minimizes $F$ (top) and $f$-moment $m_{f}$ (bottom) versus $J$ for different $\delta=2-n_{e}$. The value $t_{1}=0.0$.

(the magnitude of the $c$-like magnetization $m_{c}$ is approximately $20 \%$ of $m_{f}$ as already discussed). $\Theta$ is zero for large $J$ but then increases below a certain $J_{c}$ which depends on $\delta$. Below $J_{c}(\delta)$ the increase initially follows the mean-fieldlike form $\Theta \propto \sqrt{J_{c}-J}$ as well as $m_{f} \propto\left|J_{c}-J\right|$, whereby the increase becomes quite abrupt for $\delta \rightarrow 0$. Both $\Theta$ and

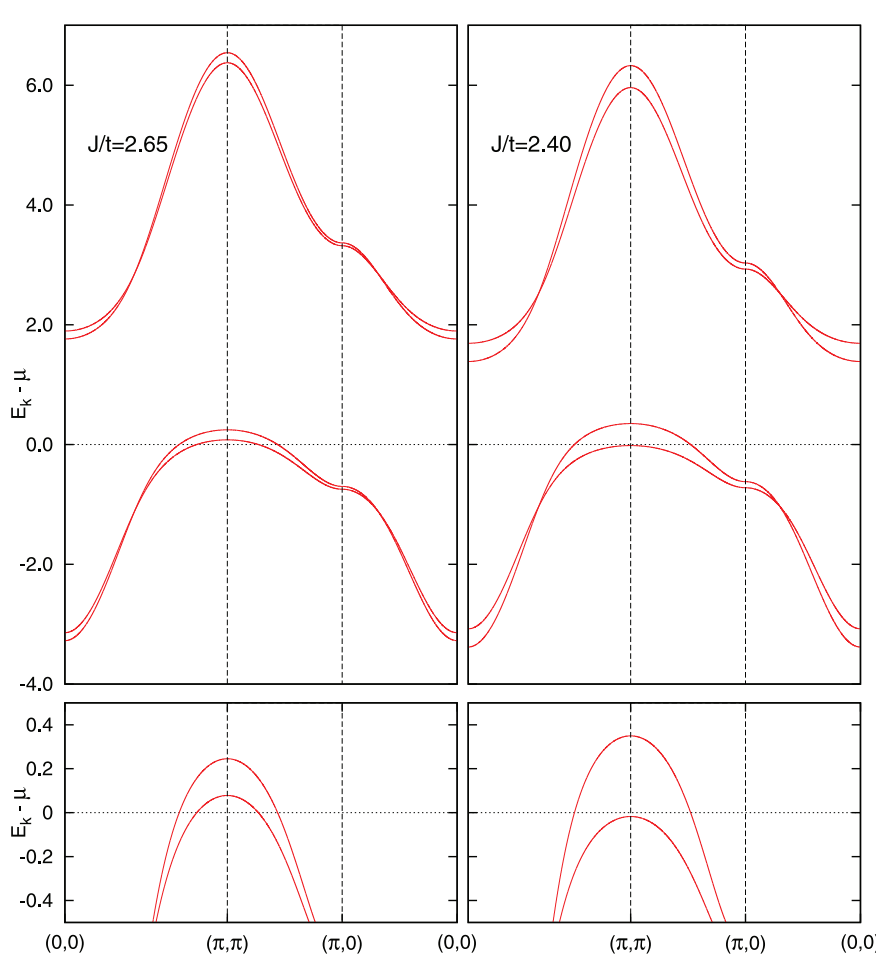

Fig. 4. Full band structure (top) and closeup around the Fermi energy (bottom) for for $J=2.65$ and $J=2.40, \delta=0.2, t_{1}=$ 0.0 .

$m_{f}$ then show a pronounced bend and increase only slowly with further decreasing $J$.

To clarify the reason for this bend, Figure 4 shows the band structure for $\delta=0.2$ and the two values $J=2.65$ and $J=2.40$, which are on opposite sides of the bend, see in Figure 3 . Below $J_{c}(\delta) \approx 2.68 \Theta$ differes from zero. The spin-degeneracy of the quasiparticle band is lifted and the Fermi surface - which takes the form of a hole-pocket around $(\pi, \pi)$ for electron densities slightly below $n_{e}=2$ - is split into two sheets. Figure 4 does show, however, that there is not just a uniform split of the two sub-bands but rather that the dispersion of the two bands is slightly different. This is precisely the deformation of the bands due to a finite value of $c_{3}$ in (9), see the discussion there. For $J=2.40$, on the other hand, the maximum of the lower band at $(\pi, \pi)$ is below $\mu$. This means that one of the two Fermi surface sheets has shrunk to a zero and only a single Fermi surface sheet remains. Figure 4 thus shows that the bend in the $\Theta$-vs.- $J$ and $m_{f}$-vs.- $J$ curves in Figure 3 corresponds to the vanishing of one Fermi surface sheet. For even smaller $J$ the Fermi surface then remains unchanged and further spin polarization can occur only via increasing polarization of the singlet background. A surprising feature is the smallness of $m_{f}$, which is much smaller than $\frac{\delta}{2}$ as expected for a fully polarized band of spin- $\frac{1}{2}$ quasiparticles. The reason is that the eigenstates of the bond Fermion Hamiltonian are not eigenstates of $S_{z}$ - see the discussion above. For example, for $J=2.4$ see the right part of Figure 4 - the weight of the first two components of $\mathbf{v}_{\mathbf{k}}$ is 0.53 in the lowest band at $\mathbf{k}=(\pi, \pi)$ and 0.47 in the second lowest band. This ratio does not 


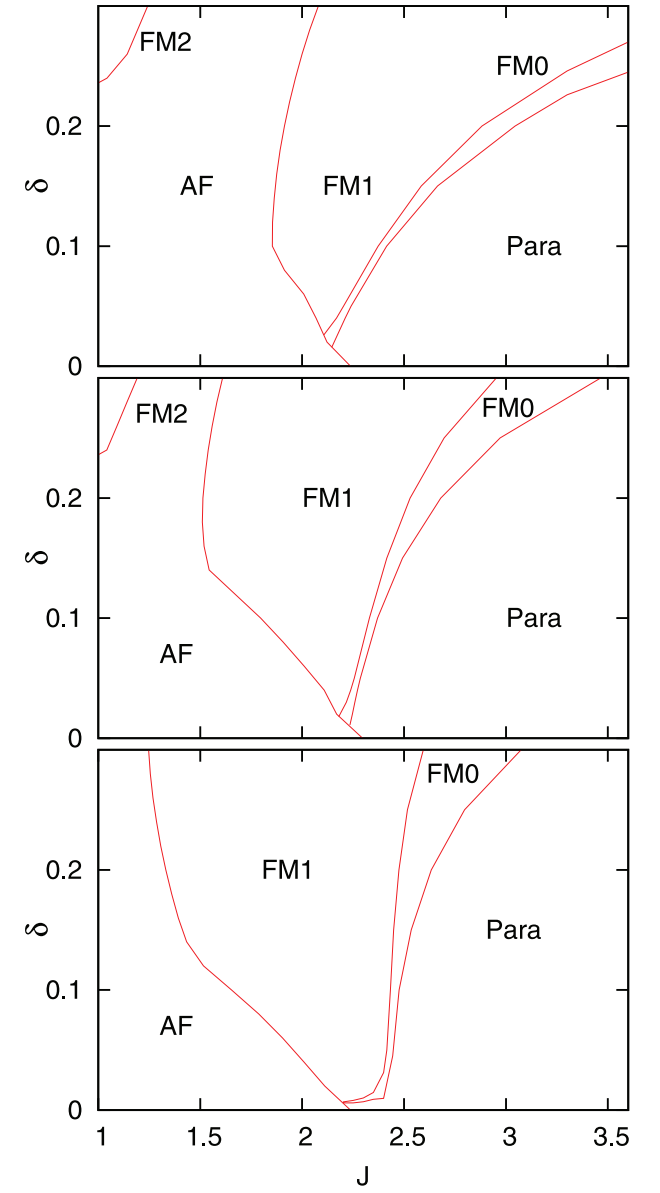

Fig. 5. Phase diagram of the Kondo lattice in the $J-\delta$ plane for $t_{1}=0.2$ (bottom), $t_{1}=0$ (center) and $t_{1}=-0.2$ (top).

change significantly. Following Kubo [29] we call the phase with the two split Fermi surface sheets FM0, the one with only a single Fermi surface sheet FM1 and the one with polarized $f$-spins (described by (18)) FM2.

Figure 5 shows the phase diagram in the $J$ - $\delta$ plane. Here we have also included the antiferromagnetic phase, which has been discussed in reference [47]. The computation of $F$ for this phase is explained in detail in this paper. For large $J$ the system is paramagnetic as could have been expected due to the dominance of the Kondo exchange term. With decreasing $J$, magnetic phases become stable for values slightly above $J=2$. Close to $\delta=0$ antiferromagnetism sets in whereas for larger $\delta$ ferromagnetic polarization is favoured. Thereby the phase FM0 - which has two heavy Fermi surface sheets - is stable only in a narrow parameter range so that the ferromagnetic phase mostly corresponds to the phase FM1 where one of the two Fermi surface sheets has shrunk to a point. The boundaries for the transitions paramagnetic $\rightarrow$ FM0 and $\mathrm{FM} 0 \rightarrow \mathrm{FM} 1$ almost merge for small $\delta$ and the point where they merge shifts to smaller $J$ as $t_{1}$ changes from positive to negative. For $t_{1}=0.2$, i.e. the lowest panel of Figure 5 , and for $2.2 \leq J \leq 2.4$ the data indicate that there is a tiny but finite range of $\delta$ where $\Theta=0$. This implies that for the Kondo insulator there is never any ferromagnetic polarization, as a function of $J / t$ there is always a phase

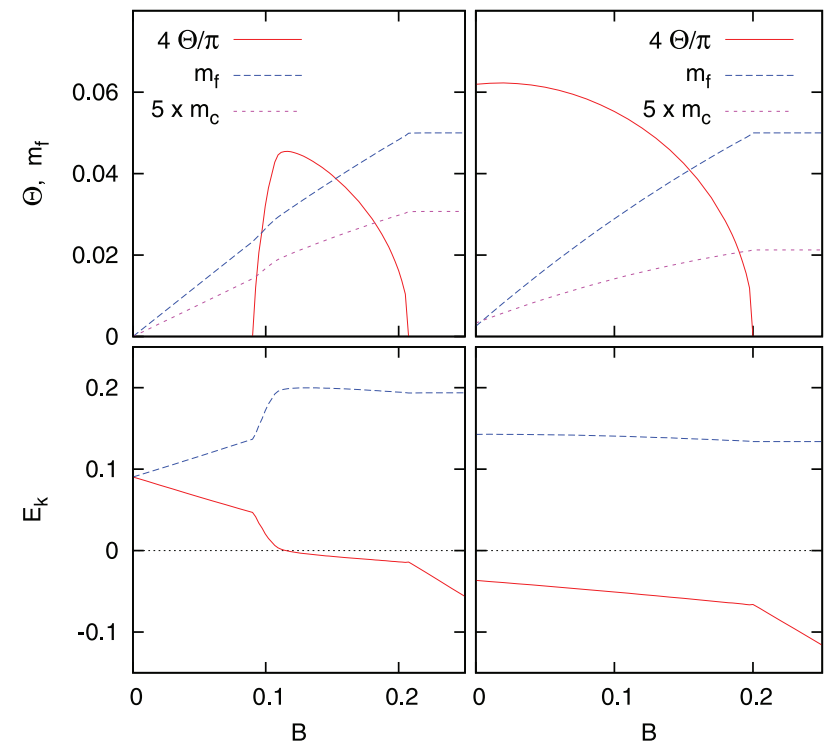

Fig. 6. Top: Angle $\Theta$ which minimizes $F$, $c$-like magnetization $m_{c}$ (multiplied by a factor of 5 ) and $f$-like magnetization $m_{f}$ versus $B$. Bottom: energies of the two lowest quasiparticle bands at $(\pi, \pi)$ versus $B$. Parameter values are $t_{1}=0.2$, $\delta=0.1$. The left part refers to $J=2.6$ the right part to $J=2.0$.

transition from the paramagnetic to the antiferromagnetic phase. Lastly, the phase FM2 which has fully polarized $f$-electrons that do not contribute to the Fermi surface volume is realized only in a small range for small $J$ and relatively large $\delta$, whereby the range increases as $t_{1}$ becomes negative. The latter is to be expected because in the phase with decoupled $f$-electrons, (18), the width of the occupied part of the $c$-electron band structure $E_{\mathbf{k}, \sigma}$ is larger for negative $t_{1}$ (see (3)). It should be kept in mind that the energy of the FM2 phase is calculated from a different and rather simplified wave function so that results concerning this phase have a qualitative nature at best.

Ferromagnetism in the KLM for electron densities in the range $n_{e}=1.5-1.8$ has been observed in numerical studies $[28,29,41-43]$ as well as in various mean-field calculations [36-40]. A ferromagnetic ground state with small magnetic moment for electron densities close to $n_{e}=2$ was found by Kubo in a VMC calculation for the periodic Anderson model in a certain parameter range [29]. We proceed to the case $B \neq 0$ but thereby disregard the antiferromagnetic phase. The possible destruction of antiferromagnetism by a magnetic field is a subtle issue which requires a more detailed discussion than is possible here. Figure 6 shows various quantities plotted versus magnetic field $B$ for $\delta=0.1$ and $t_{1}=0.2$. The left and right part of the figure refer to $J=2.6$ and $J=2.2$. Inspection of the phase diagram in Figure 5 shows that for $J=2.6$ the system is paramagnetic for $B=0$ whereas it is in the FM1 phase for $J=2.0$. The top part of the figure shows $m_{f}$ and the angle $\Theta$ as functions of $B$, the bottom parts shows the energies of the two quasiparticle bands at $(\pi, \pi)$. We first consider $J=2.6$ (left part of the figure). With increasing $B, \Theta$ initially remains zero, that means there is no polarization of the spin background, and the 
system responds to the magnetic field by the symmetric spin-splitting of the otherwise rigid quasiparicle bands, see (14). Accordingly, $m_{f}$ increases linearly with $B$. As $B$ increases, $\Theta$ takes on a small but onvanishing value leading to an abrupt increase of the splitting of the quasiparticle bands so that the lower band crosses the Fermi level and the transition FM0 $\rightarrow$ FM1 takes place. Surprisingly the slope of the magnetization versus $B$ curve increases only very little because - as already discussed above - for $\Theta \neq 0$ the quasiparticles have mixed spin- $\uparrow$ and spin- $\downarrow$ character, see the Hamiltonian (9). For even higher $B, \Theta$ becomes zero again, the magnetization saturates at $m_{f}=\frac{\delta}{2}$ and the band splitting increases linearly with $B$. The upper quasiparticle band retains its position relative to the Fermi energy which is necessary to keep the Fermi surface area constant, whereas the lower band moves away from the Fermi energy with increasing $B$. The c-like magnetization $m_{c}$ follows $m_{f}$ but is substantially smaller. This is because whereas the $f$-occupation is fixed to one per unit cell, the conduction electrons undergo charge fluctuations which will reduce any kind of ordered moment. Interestingly $m_{c}$ has the same sign as $m_{f}$ which is to be expected because the magnetic field favours ferromagnetic polarization of both, $f$-electrons and conduction electrons.

We consider $J=2.0$ (right part of the figure). There, the system is in the FM1 phase for $B=0$, i.e. the angle $\Theta$ is finite even and there is a small but finite $m_{f}$. Switching on $B$ leads to a reduction of $\Theta$, while simultaneously $m_{f}$ increases. This appears strange at first sight but reduction of $\Theta$ reduces the mixing of the $\uparrow$ - and $\downarrow$-component of the quasiparticle wave function while at the same time the magnetic field splits the two spin components. In this way, the $\downarrow$-character of the unoccupied quasiparticle states in the hole pocket around $(\pi, \pi)$ - that means the upper band in the figure - increases so that that the magnetization increases as well. Once $\Theta=0$ has been reached, $m_{f}$ again saturates at $m_{f}=\frac{\delta}{2}$. The lower part of the figure shows that during the decrease of $\Theta$ the band structure around $(\pi, \pi)$ shows very little change. The system is already in the FM1 phase for $B=0$, where only one Fermi surface sheet exists any more. The spin polarization is not achieved by a polarization of the band structure itself, but by the 'unmixing' of the two spin components in the quasiparticle wave functions.

Figure 7 shows the behaviour of the Kondo phase over a wider range of $B$. We consider the case $\delta=0.1$ and $t_{1}=0$ so that the system is in the FM1 phase for $B=0$. Increasing $B$ again leads to a reduction of $\Theta$ and an increase of the magnetization. At $B=0.17, \Theta$ reaches zero so on from this value of $B$ we have the undeformed but spin-split quasiparticle bands, whereby the $\uparrow$-band - labeled 1 in the figure - is completely filled, while the $\downarrow$-band 2 forms the hole pocket around $(\pi, \pi)$. The Fermi energy now is within the band 2 and the magnetization is constant because the size of the hole pocket is determined by the electron density. At $B=0.345$, however, there is a change in the Fermi surface in that the the $\uparrow$-quasiparticle band at $(0,0)-$ labeled 3 in the figure - crosses the Fermi energy. This results in an electron pocket around $(0,0)$ and the magnetization increases rapidly. This behaviour was also found in mean-field and DCA-calculations [43]. Figure 8 shows

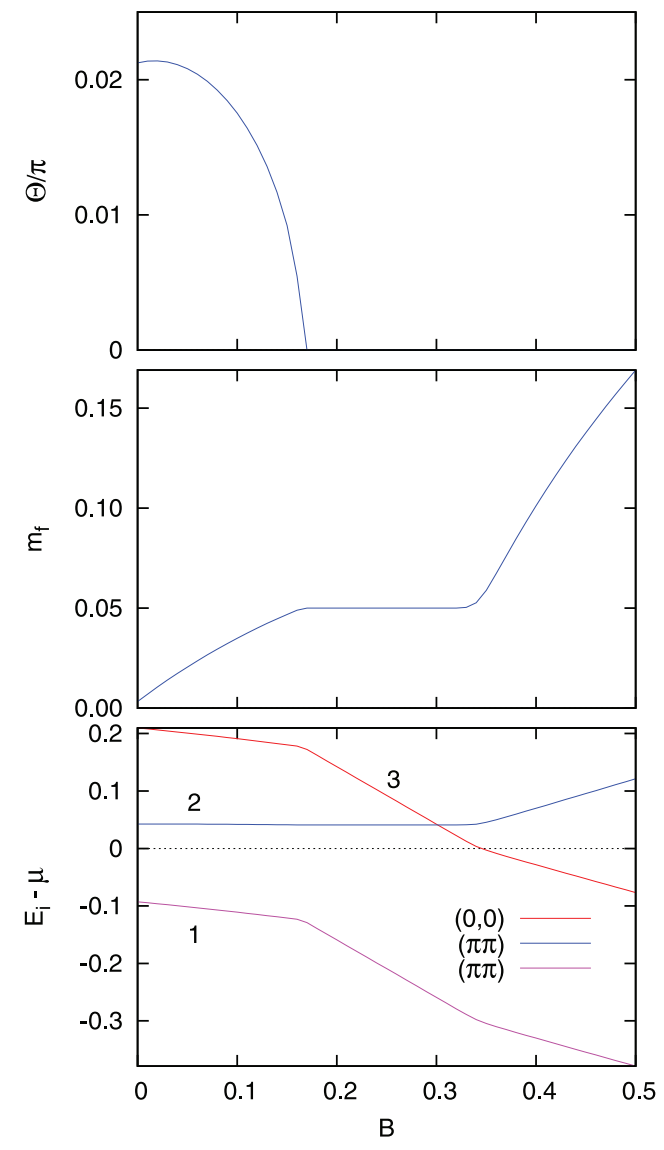

Fig. 7. Angle $\Theta$ which minimizes $F$ (top), $f$-like ordered moment $m_{f}$ (middle) and energies of the states around the Kondo gap at $\mathbf{k}=(\pi, \pi)$ and $\mathbf{k}=(0,0)$ (bottom) versus $B$. Here $J=1, \delta=0.1$ and $t_{1}=0$.

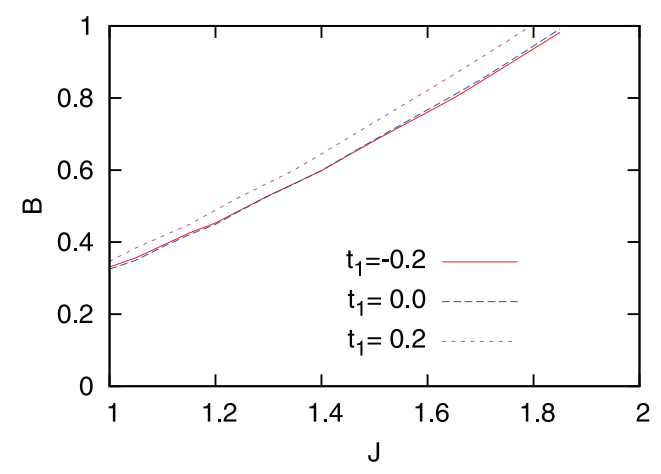

Fig. 8. Magnetic field value where the electron pocket at $\mathbf{k}=$ $(0,0)$ starts to form versus $J, \delta=0.1$.

the magnetic field where the electron pocket starts to form - i.e. where the band labeled 3 in Figure 8 crosses $\mu$ - as a function of $J$.

\section{Conclusion}

In summary we have studied ferromagnetism in the Kondo lattice model using the bond Femion method. A main 
results is that a ferromagnetic phase with a small ordered moment can be found already for electron concentrations close to $n_{e}=2$ and at similar values of $J$ where antiferromagnetism sets in. As discussed previously for the case of the antiferromagnetic phases, the bond Fermion method may be expected to give reasonable results in this range of parameters [47]. Numerical studies so far have not found evidence for this weakly ferromagnetic phase, with the possible exception of a VMC study for the PAM [29]. The weakly ferromagnetic phase may be viewed as a modification of the Kondo phase - in which the $f$-electrons do contribute to the Fermi surface volume - with triplets condensed into momentum $(0,0)$. The smallness of the ordered moment also is due to the fact that the condensed triplets lead to a mixing of the $\uparrow$ - and $\downarrow$-component in the quasiparticle wave functions. As already mentioned in the introduction, bond Fermion theory gives more of a 'rescaled version' of reality, that means the absolute values of $J$ where magnetism sets in are not reproduced correctly. The exact value where antiferromagnetism sets in for $n_{e}=2$ at $t_{1}=0$ is $J_{c}=1.45$ [23], whereas from Figure 5 one would estimate $J_{c}=2.3$. It was shown in reference [47] that when the phase diagram for the antiferromagnetic phases is plotted in the $\left(J / J_{c}, n_{e}\right)$-plane rather than the $\left(J, n_{e}\right)$-plane, good agreement with VMC could be achieved and one might expect the same to hold true for the phase diagrams in Figure 5.

In the present study we did not consider incommensurate magnetic order which might win over the ferromagnetic phase. On the other hand, in systems with easy-axis-like magnetic anisotropy such incommensurate phases may be suppressed and the weakly ferromagnetic phase be indeed observable.

Open access funding provided by Projekt DEAL.

\section{Author contribution statement}

All authors contributed equally to the research presented in this paper.

Publisher's Note The EPJ Publishers remain neutral with regard to jurisdictional claims in published maps and institutional affiliations.

Open Access This is an open access article distributed under the terms of the Creative Commons Attribution License (http://creativecommons.org/licenses/by/4.0), which permits unrestricted use, distribution, and reproduction in any medium, provided the original work is properly cited.

\section{References}

1. G.R. Stewart, Rev. Mod. Phys. 56, 755 (1984)

2. P.A. Lee, T.M. Rice, J.W. Serene, L.J. Sham, J.W. Wilkins, Commun. Condens. Matter Phys. 12, 99 (1986)

3. G.R. Stewart, Rev. Mod. Phys. 73, 797 (2001)
4. H.v. Löhneysen, A. Rosch, M. Vojta, P. Wölfle, Rev. Mod. Phys. 79, 1015 (2007)

5. Q. Si, F. Steglich, Science 329, 1161 (2010)

6. J.R. Schrieffer, P.A. Wolff, Phys. Rev. 149, 491 (1966)

7. A. Yoshimori, A. Sakurai, Progr. Theor. Phys. Supp. 46, $162(1970)$

8. C. Lacroix, M. Cyrot, Phys. Rev. B 20, 1969 (1979)

9. C. Lacroix, J. Magn. Magn. Mater. 100, 90 (1991)

10. A. Auerbach, K. Levin, Phys. Rev. Lett. 57, 877 (1986)

11. S. Burdin, A. Georges, D.R. Grempel, Phys. Rev. Lett. 85, 1048 (2000)

12. G.-M. Zhang, L. Yu, Phys. Rev. B 62, 76 (2000)

13. M. Lavagna, C. Pepin, Phys. Rev. B 62, 6450 (2000)

14. T. Senthil, M. Vojta, S. Sachdev, Phys. Rev. B 69, 035111 (2004)

15. M. Vojta, Phys. Rev. B 78, 125109 (2008)

16. G.-M. Zhang, Y.-H. Su, L. Yu, Phys. Rev. B 83, 033102 (2011)

17. J. Nilsson, Phys. Rev. B 83, 235103 (2011)

18. C.C. Yu, S.R. White, Phys. Rev. Lett. 71, 3866 (1993)

19. S. Moukouri, L.G. Caron, Phys. Rev. B 52, 15723(R) (1995)

20. S. Moukouri, L.G. Caron, Phys. Rev. B 54, 12212 (1996)

21. T. Mutou, N. Shibata, K. Ueda, Phys. Rev. Lett. 81, 4939 (1998)

22. S. Smerat, U. Schollwock, I.P. McCulloch, H. Schoeller, Phys. Rev. B 79, 235107 (2009)

23. F.F. Assaad, Phys. Rev. Lett. 83, 796 (1999)

24. Z.-P. Shi, R.R.P. Singh, M.P. Gelfand, Z. Wang, Phys. Rev. B 51, 15630(R) (1995)

25. W. Zheng, J. Oitmaa, Phys. Rev. B 67, 214406 (2003)

26. H. Watanabe, M. Ogata, Phys. Rev. Lett. 99, 136401 (2007)

27. M.Z. Asadzadeh, F. Becca, M. Fabrizio, Phys. Rev. B 87, 205144 (2013)

28. K. Kubo, Phys. Rev. B 87, 195127 (2013)

29. K. Kubo, J. Phys. Soc. Jpn. 84, 094702 (2015)

30. R. Peters, N. Kawakami, Phys. Rev. B 92, 075103 (2015)

31. L.C. Martin, F.F. Assaad, Phys. Rev. Lett. 101, 066404 (2008)

32. L.C. Martin, M. Bercx, F.F. Assaad, Phys. Rev. B 82, 245105 (2010)

33. D. Aoki, J. Flouquet, J. Phys. Soc. Jpn. 81, 011003 (2012)

34. D. Aoki, T. Combier, V. Taufour, T.D. Matsuda, G. Knebel, H. Kotegawa, J. Flouquet, J. Phys. Soc. Jpn. 80, 094711 (2011)

35. M. Brando, D. Belitz, F.M. Grosche, T.R. Kirkpatrick, Rev. Mod. Phys. 88, 025006 (2016)

36. S.V. Kusminskiy, K.S.D. Beach, A.H. Castro Neto, D.K. Campbell, Phys. Rev. B 77, 094419 (2008)

37. O. Howczak, J. Spałek, J. Phys.: Condens. Matter 24 $205602(2012)$

38. O. Howczak, J. Kaczmarczyk, J. Spałek, Phys. Stat. Solidi B 250, 609 (2013)

39. M.M. Wysokiński, M. Abram, J. Spałek, Phys. Rev. B 90, 081114(R) (2014)

40. M.M. Wysokiński, M. Abram, J. Spałek, Phys. Rev. B 91, 081108(R) (2015)

41. K.S.D. Beach, F.F. Assaad, Phys. Rev. B 77, 205123 (2008)

42. R. Peters, N. Kawakami, T. Pruschke, Phys. Rev. Lett. 108, 086402 (2012)

43. M. Bercx, F.F. Assaad, Phys. Rev. B 86, 075108 (2012) 
44. R. Eder, O. Stoica, G.A. Sawatzky, Phys. Rev. B 55, 47. R. Eder, K. Grube, P. Wróbel, Phys. Rev. B 93, 165111 R6109 (1997) (2016)

45. R. Eder, O. Rogojanu, G.A. Sawatzky, Phys. Rev. B 58, 7599 (1998)

46. C. Jurecka, W. Brenig, Phys. Rev. B 64, 092406 (2001)

48. R. Eder, P. Wróbel, Phys. Rev. B 98, 245125 (2018)

49. S. Sachdev, R.N. Bhatt, Phys. Rev. B 41, 9323 (1990) 